\title{
20. Spotlight: Natur, Kultur und Covid-19 - ein Essay für eine globale Seuchengeschichte
}

Covid-19 hat die Welt im Griff. Covid-19 wird durch ein als SARS-CoV-2 benanntes Virus verursacht. Die klinischen Symptome sind besonders im Frühstadium nicht ausreichend spezifisch. Deshalb dient der Virusnachweis mittels Polymerase Kettenreaktion $(\mathrm{PCR})^{1}$ in den Worten des Robert-Koch-Institutes als „Goldstandard“, um die klinische Diagnose zu sichern. ${ }^{2}$ Heutzutage ist die Virologie von der Diagnostik bis zur Therapie eine molekularbiologische Wissenschaft, in der die Gentechnologie eine entscheidende Rolle spielt. Auch in der Seuchenbekämpfung, die auf Erregernachweis und Isolierung des Erregers bzw. seiner Träger/-innen setzen muss, ist es folgerichtig, diagnostische Evidenzen - im klassischen Sinne des unmittelbar Einleuchtenden (Martin/Fangerau, 2021) - mit molekulargenetischen Verfahren zu erzeugen. Dies gilt ganz und gar für die Entwicklung neuer Impfseren (zur Corona-Impfstoffentwicklung siehe Korte, Kap. 19) wie offenkundig der mRNA-Vakzine - und neuer spezifischer Heilmittel.

Eine rein molekularbiologische Sichtweise samt der entsprechenden Technologie gerät aber an ihre Grenzen, wenn Pandemien, ihr Erleben und ihre Folgen in einer breiteren Perspektive betrachtet werden. Intensivmediziner/-innen, Psychiater/-innen, Soziologen/Soziologinnen, Epidemiologen/Epidemiologinnen oder Ökonomen/Ökonominnen sowie vor allem die breite Öffentlichkeit richten ihr Augenmerk auf gänzlich andere Aspekte des Seuchengeschehens als sie das Molekulare bietet und kommen daher zu anderen Folgerungen, wie mit der Seuche und folglich auch mit dem Virus umzugehen ist. Der genetische Virusnachweis rückt für sie in den Hintergrund oder ist ggf. sogar irrelevant. Paradigmatisch steht hier die spätestens seit September 2020

1 Die PCR ist eine Möglichkeit, spezifisch bestimmte Abschnitte eines genetischen Materials zu vervielfältigen und somit nachweisbar zu machen.

2 Siehe unter: https://www.rki.de/DE/Content/InfAZ/N/Neuartiges_Coronavirus/Vorl_ Testung_nCoV.html;jsessionid=149C2C54C9D26381AF48C8F6C2695729.internet081?nn=13490888\#doc13490982bodyText4 [27.02.2021]. 
geführte Debatte, ob Menschen an oder mit dem Coronavirus sterben (Viglione, 2020). Hinter dieser Frage steht die alltagsweltliche Forderung, die reine und wenig aussagekräftige Ziffer von Coronatoten zu differenzieren; ferner sollen der reine Virusnachweis und die dazu genutzten Methoden in einen gesellschaftlichen Zusammenhang gestellt werden.

Auch Historiker/-innen und Medizinhistoriker/-innen haben sich vielfach und vielerorts in unterschiedlichen Medien zur aktuellen Pandemie geäußert. In Zeiten von Pandemien gilt die Geschichte als Sinngeberin: der Blick darauf, wie frühere Zeiten mit Seuchen umgegangen sind, hilft bedrohliche Unwägbarkeiten, bedrohliche Kontingenzen zu reduzieren. In diesem Sinne möchten wir in diesem Essay für neue Ansätze in der Seuchengeschichte plädieren, um einen zwar multiperspektivischen, aber zugleich integrativen Blick auf das Seuchengeschehen zu richten, so wie es für die Gegenwart gefordert wird.

Grundlage unserer Gedanken ist, dass die Geschichte von Seuchen notwendig aus verschiedenen Dimensionen betrachtet werden muss. Auf dieser Basis wollen wir die Idee eines globalen und vernetzten Zugangs zur Geschichte der Seuchen ins Zentrum rücken. Wegweisend für unsere Gedanken sind das Verhältnis von Natur und Kultur, der Austausch und Verkehr zwischen den verschiedenen Räumen und dies auf der Basis der sich stets wandelnden Grundlagen von Produktion und Reproduktion. Der weltweite Zusammenhang von Natur, Kultur und modernen Seuchen sowie der dazu passende methodische Ansatz nahtloser Netzwerke bilden den Schluss und das Fazit unserer Gedanken und Vorschläge.

\subsection{Integrierte Ansätze und ein neuer Blick auf die Geschichte angesichts einer gegenwärtigen pandemischen Krise}

In einer Zeit, in der scheinbar sichere Annahmen ständig revidiert werden müssen, erscheint die Geschichte als ein Hort historisch geronnener Handlungsoptionen: Historische Erfahrungen bilden die Grundlage unseres heutigen Gesundheitswesens (Fangerau/Labisch, 2020). Der genetische Virentest ist dabei ein epistemologisch zentrales und anregendes, historisch aber nur bedingt relevantes Verfahren. Zum Verständnis des gesellschaftlichen Umgangs mit früheren Seuchen trägt ein molekularbiologischer Nachweis - wie übrigens alle modernen Nachweise von Krankheitserregern - nur wenig bei: dies muss hier deshalb deutlich gemacht werden, weil gelegentlich der Anspruch erhoben wird, die Geschichtsschreibung paläopathologisch und molekulargenetisch 
revolutionieren zu wollen (Meier, 2020; Mordechai et al., 2020). Ein Beispiel: die vor einigen Jahren erfolgte biologische Identifikation des Erregers des „Schwarzen Todes“ (Spyrou et al., 2016) hilft nur höchst bedingt, die Geschichte dieser säkularen Seuche in ihrem unmittelbaren Wirken und ihren langfristigen Folgen zu verstehen (dazu Bergdolt, 1989 und 1994). Der retrospektive Beweis spezifischer Krankheitserreger ist lediglich ein Baustein unter vielen anderen, um frühere Infektionskrankheiten und Seuchen in ihrem gesamten Ausmaß zu erfassen (Labisch, 2018). Und diese Grenzen biologischer oder medizinischer Aussagen gelten notwendig auch für das aktuelle Seuchengeschehen.

Zwischen biologischem Virusnachweis und kultureller Reaktion scheint somit aktuell wie historisch ein Graben zu klaffen: Die Biologie und das Virus als natur- bzw. lebenswissenschaftlich essentialisierte Entität stehen als unbeherrschte Natur auf der einen, die Menschen und Gesellschaften stehen mit ihrer Kultur, ihrem Sozialleben und den dadurch definierten Handlungsspielräumen auf der anderen Seite. Diese Handlungsspielräume wiederum lassen sich weiter in Systeme wie Politik, Wissenschaft, Technik oder Kultur ausdifferenzieren, die wiederum unabhängig voneinander betrachtet werden können.

Indes reicht eine solche Trennung von diversen menschlichen Handlungsspielräumen und naturbedingten Kontexten weder für eine historische noch für eine soziologische Analyse aus, und zwar nicht einmal dann, wenn sich die Geschichtsschreibung oder die Soziologie auf den Bericht über eines dieser Systeme beschränken. Darauf haben seit einem Jahrhundert gerade Medizinhistoriker/-innen oder Philosophen/Philosophinnen immer wieder hingewiesen. Der Ansatz von Ludwik Fleck, Wissenschaft als sozial bestimmte, disziplinierte Interpretation von beobachteter Umwelt aufzufassen (Fleck, 1980 [1935]), steht hier ebenso exemplarisch wie das Angebot von Bruno Latour, die Mobilisierung und Beschreibung der Welt als Zusammenspiel menschlicher und dinglicher Handlungen und Handlungsangebote zu begreifen (Latour, 1995 und 2002).

Soweit wir die durch SARS-CoV-2 bzw. Covid-19 ausgelöste Pandemie bislang überhaupt verstehen, handelt es sich in vielerlei Hinsicht um ein teils zwar bekanntes, teils aber auch neues Geschehen, das nur aus einer integrativen Perspektive erfasst werden kann. So weist die aktuelle Pandemie in ihrem Ablauf, ihrer gesellschaftlichen Verarbeitung, ihren sozialen Konsequenzen, ihrer politischen Kommunikation und medizinischen Antwort viele Parallelen zu historischen Pandemien auf. Gleichzeitig trifft sie auf eine völlig andere Gesellschaft in einer anders vernetzten Welt als vergangene, vergleichbare Pandemien. Nicht nur sind Raum und Zeit seit z. B. der Poliopandemie der 1950er Jahre - metaphorisch gesprochen - weiter geschrumpft. Auch die veränderten politischen Verhältnisse oder die neuen Möglichkeiten der digitalen Kommunika- 
tion und Datenverarbeitung (siehe Winkler/Prainsack, Kap. 17, zu Big Data in der personalisierten Medizin) tragen zu einer Andersartigkeit der heutigen Pandemien bei. Diese auf den ersten Blick wenig revolutionäre Erkenntnis ermöglicht es aber der Geschichtsschreibung, auf die Geschichte von anderen Seuchen einen neuen und vielleicht anderen Blick zu werfen, um den gesamten Ereignisraum von den Ursachen, unter denen neue Erreger entstehen, über die weltweite Ausbreitung bis hin zu den Auswirkungen vor Ort zu erfassen.

\subsection{Natur, Kultur und ökologische Räume}

Den ersten Ansatz bzw. die Grundlage für eine integrative und damit getrennte Perspektiven wieder zusammenführende Geschichte von Seuchen bildet der Wirkzusammenhang von Natur und Kultur. Für eine solche Sichtweise ist ein interpretativer Kultur- bzw. Naturbegriff hilfreich, der Kultur nicht als Gegenstück von Natur und vice versa begreift, sondern die zeitlich gebundene jeweilige Interpretation dessen, was als Natur und Kultur begriffen wird, in den Mittelpunkt rückt. In ein solches Verständnis eingeschlossen ist die Idee, dass die Menschen zwar selbst Subjekte der Natur sind, ihnen die Natur zugleich aber als Objekt gegenübersteht. Menschen interpretieren diese Natur als das Andere, nicht von ihnen Gemachte. Dabei übersehen die Menschen, dass sie diese Natur im Moment des Kontaktes auch über die Distanz hin schon benannt und damit eingeordnet und für den menschlichen Handlungsraum erschlossen haben. Auf den Prozess, dasjenige zu erschließen, zu interpretieren und letztlich zu benennen, was Menschen als Krankheit begreifen, wirken Kultur und Gesellschaft ein. Charles Rosenberg hat für dieses Vorgehen den Begriff des „framing“ vorgeschlagen. Krankheiten können demnach nicht als rein biologische, als rein kulturelle oder als rein soziale Konstrukte gesehen werden. Vielmehr greifen im Prozess des Deutens und des Definierens, des Eingrenzens eines bestimmten Namens (Benamung), Natur und Kultur ineinander (Rosenberg, 1989 und 1992). Kognition, Emotionen, Körpersensationen und kollektive kulturelle Konventionen interagieren bei der Entwicklung von Krankheitskonzepten (Fangerau et al., 2009).

Diese anthropologische Erzählung von einer Natur, deren Sein und Benamung vom Menschen abhängt, muss für eine historische Untersuchung handhabbar gemacht werden. Hierzu kann das Modell ökologisch ausgeglichener Räume dienen: In diesen Räumen besteht ein labiles Gleichgewicht zwischen Natur und Kultur einschließlich sämtlicher tierischer - selbstredend inklusive menschlicher - und pflanzlicher Lebewesen samt den bekannten und durchlebten, damit gleichsam „enkulturierten“ Krankheiten. „Enkulturiert“ meint in diesem Zusammenhang, dass die Menschen von der Geburt an 
zunächst unbewusst, dann bewusst in bestimmte Deutungs- und Handlungsmuster und damit eben auch in kulturspezifische Deutungen von Gesundheit und Krankheit - hineinwachsen. George Rosen schrieb in diesem Sinne 1947:

Gesundheit und Krankheit sind Ausdruck einer sich verändernden Beziehung zwischen den verschiedenen Teilen des Körpers sowie zwischen dem Körper und der äußeren Umgebung, in der er ,ist‘. Als biologisches Phänomen werden die Ursachen von Krankheiten im Bereich der Natur gesucht, aber beim Menschen hat Krankheit noch eine andere Dimension. Nirgends erscheint menschliche Krankheit als ,reine Natur; vielmehr werden Krankheiten immer durch soziale Aktivität und die durch sie geschaffene kulturelle Umgebung vermittelt und modifiziert. (Rosen, 1947) ${ }^{3}$

Diese Austausch-, Vermittlungs- und Aushandlungsbeziehung zwischen Natur, sozialer Aktivität und kultureller Umgebung lässt sich bildlich mit dem Konzept von Biosphären beschreiben. So wie unterschiedliche Lebensräume aufeinander einwirken und sich gegenseitig beeinflussen, so stellt sich eine Form von Gleichgewicht zwischen Krankheit als „reiner Natur“ sowie ihren sozialen und kulturellen Deutungsräumen ein. Das Konzept ausgeglichener Biosphären orientiert sich an allen Floren und Faunen (z. B. invasive Pflanzen- und Tierarten), die sämtlich in einem regen und ggf. folgenschweren Austausch stehen.

Auf Basis eines solchen Ansatzes lassen sich folgende Szenarien für Seuchengeschehen von ihrem Ursprung bis zu ihrer Ausbreitung aufstellen:

- Das - immer labile - ökologische Gleichgewicht wird von innen heraus gestört - beispielsweise durch urbane Verdichtungen, durch Ausweiten der Landwirtschaft und gemeinsame Tierhaltung verschiedener Arten etc. (Beispiele bieten neue urbane Zonen, z. B. in China, mit massiven Problemen der Ver- und Entsorgung; Eindringen der Landwirtschaft in (Ur-)Wälder in tropischen Zonen Afrikas oder Brasiliens; Verschieben der Grenzen des Zusammenlebens von Mensch und Tier).

- In ökologisch ausgeglichene Räume dringen Menschen ein, die mit den ebendort enkulturierten Krankheiten bis dahin keinen Kontakt hatten (Beispiele bieten orts-

3 Übersetzt durch die Autoren. Original: „Health and disease are expressions of changing relationships between the various components of the body, and between the body and the external environment in which it has its being. As a biological phenomenon, the causes of disease are sought in the realm of nature; but in man disease has still another dimension. Nowhere does human disease occur as , pure nature'; instead it is ever mediated and modified by social activity and the cultural environment which such activity creates." 
gebundene typische Krankheitserreger [z. B.: Typhus; Malaria] oder die Invasion der Europäer in verschiedenen Phasen der Kolonialisierung; migrierende Bevölkerungen).

- Krankheitserreger werden in andere ökologische Zonen getragen und äußern sich hier in einzelnen Infektionen, in Epi- und möglicherweise in Pandemien (dies können bekannte Erreger sein, wie z. B. Cholera, oder bis dato unbekannte Erreger, die so genannten „new emerging diseases“ etc., beides bei unvorbereiteten Immunsystemen als ,virgin soil epidemics“ besonders ausgeprägt).

Kommt es in einer Gesellschaft zu bis dahin unbekannten Krankheitsphänomenen, wird in einem gesellschaftlichen Aushandlungsprozess eine neue Krankheit definiert. Mit dem Erstarken einer technologisch ausgerichteten Medizin, deren Konzeption darin besteht, dass das Körpergeschehen naturwissenschaftlich erklärt und technisch kontrolliert werden kann, ${ }^{4}$ spielen Naturwissenschaften und Medizin in dem Prozess des Aushandelns eine immer bedeutendere, wenngleich niemals die einzige Rolle. Die eingangs geschilderte molekulargenetische Definitionsmacht über den Virusnachweis bietet ein aktuelles Beispiel. Schließlich veranlasst - wiederum als Beispiel - SARSCoV-2 als benamte Natur die Menschen zu Gegenreaktionen, die tief gesellschaftlich verwurzelt sind. Verschiedene Kulturen und Zivilisationen handeln natürlich - sprich: selbstverständlich ${ }^{5}$ - unterschiedlich.

Bei allen Zusammenhangsgeschichten sollte indes eines deutlich sein: Ein Gleichgewicht zwischen den Sphären der außerhalb des Menschen stehenden Natur, seiner als Biologie begriffenen Verfasstheit sowie dem menschlichen sozialen und kulturellen Handeln ist nie stabil, sondern muss stets neu ausgeglichen werden. Selbst dann, wenn eine Krankheit als „besiegt“ erscheint, ist das nur eine Momentaufnahme in einem langfristigen Versuch, ein Gleichgewicht zwischen Natur und Mensch zu finden. So würden die angeblich beherrschten Pocken bzw. viele angeblich beherrschte endemische Krankheiten wieder virulent, falls die Erreger freigesetzt würden - wie die in Atlanta oder Novosibirsk gelagerten Pockenviren - oder falls die Krankheitserreger Resistenzen entwickeln, wie etwa die methycillinresistenten Staphylococcen, die für die gefürchteten nosokomialen (im Krankenhaus auftretenden) Infektionen verantwortlich sind.

$4 \quad$ Zu Konzepten der Medizin vgl. grundlegend Karl Eduard Rothschuh (1978).

5 Arnold Gehlen weist in seiner Institutionenlehre darauf hin, dass der in gesellschaftlichen Bezügen umgangssprachlich verwandte Begriff „,natürlich“ nicht „Natur“ meint, sondern das gesellschaftlich und damit „selbstverständlich“ Gegebene, dem gleichsam eine „natürliche“ Notwendigkeit zugeordnet wird; vgl. u. a. Gehlen (1956). 
Ein solcher Zugang, der das Mensch-Natur-Verhältnis als unauflöslichen Wirkzusammenhang versteht, bietet einen möglichen Einstieg in die Seuchengeschichte.

\subsection{Verkehr}

Der zweite Ansatz, den es in neuer Weise zu beachten gilt, fokussiert den Verkehr von Menschen und Waren in seinem historischen Wandel. Die bisherige Historiographie bietet hier bekannte Ausgangspunkte: die großen historischen Seuchenzüge - vom "loimos“ (altgriechisch für „Pestilenz“, „Seuche“) eines Thukydides 430 BCE in Athen über die Antoninische ( $(\mathrm{b} 165 \mathrm{CE})$, die Cyprianische (ab $250 \mathrm{CE}$ ) oder die Iustinianische Pest (ab ca. 6. Jh. CE) - haben sich schon immer über Handelswege verbreitet. Dies ist von Jahr zu Jahr nachzuverfolgen am unaufhaltsamen Voranschreiten des „großen Sterbens“, das als "Schwarzer Tod“ seit 1330 CE von Zentralasien aus seinen Weg nahm. Aber erst die im Zuge der Eroberung der Welt permanent anwachsenden globalen Handelsnetze haben zu einem „Columbian Exchange“ (Crosby, 1972) und schließlich zu einer „unification microbiénne du monde“ geführt (Le Roy Ladurie, 1973): Denn die „Entdeckung Amerikas“ führte dazu, dass nicht nur Menschen, sondern auch Tiere, Pflanzen und eben auch Krankheiten und vieles andere mehr zwischen den Kontinenten ausgetauscht wurden - und dies wiederum hatte zur Folge, dass letztlich die ganze Welt mikrobiell vereinheitlicht und so in ihren Krankheitserregern vereint wurde.

Tatsächlich besteht dieser Zusammenhang zwischen Verkehr und Seuchen, soweit sich die Geschichte zurückverfolgen lässt (McNeill, 1976) - und ist darüber hinaus bereits in den unterschiedlichen Kulturräumen der Erde angelegt (Diamond, 1997). Erst in Zeiten der Industrialisierung und des Imperialismus und der damit einhergehenden „Verwandlung der Welt“ (Osterhammel, 2009) kam es mit dem weltweiten zügigen Austausch von Massengütern und Menschenmassen auch zu einem regelmäßigen weltweiten Austausch von Krankheitserregern - seinerzeit maßgeblich durch den Schiffsverkehr. Die „skandalisierten“ Seuchen der Industrialisierung wurden die pandemischen Züge der Cholera. „Skandalisiert“ heißt, dass die öffentliche Wahrnehmung, die Diskussion und die Folgen einer Krankheit oder Seuche in einem eklatanten Missverhältnis zu ihrer tatsächlich vergleichsweise geringen epidemiologischen Bedeutung stehen. Jedoch haben die damaligen Wissenschaftler/-innen und Politiker/-innen angesichts der Frage „Absperrung“ oder "freier Verkehr“ mit langfristigen und unvorstellbar teuren Maßnahmen dafür gesorgt, dass die Cholera auf ihre klassischen endemischen (regional beschränkten) Bereiche begrenzt wurde und durch entsprechende Maßnahmen weiterhin begrenzt wird. Der weltweit freie Verkehr von Menschen, Waren und Diensten war so sichergestellt. Die Industrialisierung wurde so die erste Hoch-Zeit der 
Denkfigur des „homo hygienicus“, in der sich ein medizinisch gedeuteter Anspruch an ein gesundes Verhalten und an gesundheitsgerechte Verhältnisse auch weltweit durchsetzte (Labisch, 1992).

Austausch und Verkehr, menschliche Reaktion und wirtschaftliche Überlegungen bieten also einen etablierten Zugang zur Seuchengeschichte, der aber in neuen Konstellationen mit dem Blick auf Natur, Kultur und ökologische Räume zu verbinden ist.

\subsection{Technische Mittel der Produktion und Reproduktion}

Vor dem Hintergrund von Natur und Kultur sowie von Austausch und Verkehr bilden die jeweils zeitgemäßen Bedingungen von Produktion und Reproduktion einen dritten zentralen Baustein für eine integrative Seuchengeschichte.

Die materielle Grundlage der aktuellen Produktionsweise sind automatisierte Techniken und die Datenverarbeitung. So wie Kohle und Dampf im 19. und 20. Jahrhundert die vormals begrenzte animalische Kraft ins Unermessliche steigerten, erweitert die Datenverarbeitung seit Ende des 20. Jahrhunderts die Rechen- und in Anfängen sogar die intelligenten Hirnleistungen des Menschen: Gentechnik ist ohne Datenverarbeitung undenkbar (zu genetischen Daten im Kontext der Gendiagnostik siehe Mundlos, Kap. 4; zum Kontext somatische Gentherapie siehe Fehse, Kap. 6). Die Globalisierung einer die gesamte Welt umspannenden Konnektivität von Rechnern, Servern und Netzwerken in sämtlichen Produktions- und Reproduktionsbereichen schreitet unaufhaltsam voran. Damit geht einher, dass sich die Menschen über die gesamte Welt ausbreiten und so bis dahin ausgeglichene Biosphären permanent herausfordern und durch den regen internationalen Verkehr miteinander verbinden.

Jüngst hat der Historiker Mark Honigsbaum auf der Basis seiner Intepretation des Zusammenspiels von Natur und Kultur gezeigt, wie Seuchen - darunter Influenza, Legionärskrankheit/Sick Building Syndrom, Aids, SARS, Ebola, Zika und Covid-19 - jeweils in der Auseinandersetzung von Politik, Wirtschaft, Medien, Sprache und nicht zuletzt auch Wissenschaft und Medizin (samt ihren Produktionsmitteln) zunächst wahrgenommen und in einem gesellschaftlichen Diskussionsprozess behandelt werden (Honigsbaum, 2020; Matthews, 2020). Dabei macht nicht etwa das Virus, was es will, es tritt aber als ein unabweislicher Akteur in Erscheinung, auf den reagiert werden muss: Der neue Akteur wird möglichst eliminiert oder es wird wie bei anderen Krankheiten, z. B. Influenza, ein neues Gleichgewicht im Sinne einer Akzeptanz des Erregers und der durch ihn hervorgerufenen Todesfälle hergestellt und toleriert.

Kulturell geprägte Kommunikationsweisen und -mittel bedingen also, was von dem Virus in welcher Weise die Wahrnehmungsschwelle überschreitet, ob also am Ende das 
Virus oder doch eine mit ihm einhergehende Erscheinung in das Zentrum der Diskussion gerät und gleichsam skandalisiert wird.

\subsection{Globale Geschichte}

Auf das gestörte Gleichgewicht von Natur und Kultur unter dem Aspekt der Globalisierung ist zuerst von Laurie Garrett 1994 mit „The Coming Plague: Newly Emerging Diseases in a World out of Balance“ hingewiesen worden. Andere - etwa S. Harris Ali und Roger Keil (Ali/Keil, 2008) oder David Quammen (2012) - setzten diese Reihe fort. Die hier angerissenen Beispiele schließen an diese Arbeiten an und zeigen, dass es weniger darauf ankommt, eine Konzeptgeschichte, Wirtschaftsgeschichte, Politikgeschichte, Sozialgeschichte oder Institutionengeschichte der Seuchen oder der öffentlichen Gesundheitssicherung zu schreiben. Denn alle diese Bereiche hängen nicht nur zusammen, sondern sie interagieren miteinander.

Marc Harrison (2015) schlussfolgert in einem energischen Plädoyer für einen globalen Ansatz in der Seuchengeschichte, dass

global über Krankheit zu denken mehr bedeutet, als die Bewegungen von Pathogenen oder sogar relativer Immunität zu betrachten. Krankheit ist ein kraftvoller Agent des Wandels, aber sie wirkt nie in einem Vakuum und es ist zu einfach, sie als einen Treiber der Globalisierung zu betrachten.

Es ist notwendig, die Dynamiken zu identifizieren, die pathogene Verbindungen hervorbringen und die dabei gleichzeitig die sozialen und natürlichen Umwelten verändern, in die Pathogene und Vektoren eingeführt werden. Nur so können wir die unterschiedlichen Auswirkungen von Krankheiten und ihre langfristigen Folgen beurteilen. ${ }^{6}$

Es gibt also vor dem generellen Hintergrund von Natur und Kultur essenzielle, zusammenwirkende Zugangswege für eine globale Seuchengeschichte:

6 Übersetzt durch die Autoren. Original: „[...] that thinking globally about disease means more than considering the movement of pathogens or even relative immunity. Disease is a powerful agent of change, but it never operates in a vacuum and to regard it as a force of globalization is simplistic. It is necessary to identify the dynamics that forge pathogenic connections and that simultaneously transform the social and natural ecologies into which pathogens and vectors are introduced. Only in this way can we account for the differential impact of disease and its longer term consequences." 
- Das dynamische Verhältnis von Natur und Kultur ist die (meta-)theoretische Grundlage. Veränderungen in lokalen und regionalen Biosphären und der Austausch zwischen Biosphären sind in einem globalen Zusammenhang empirisch zu erheben.

- Der sozial und kulturell bedingte wissenschaftliche und gesellschaftliche Aushandlungsprozess, der zur Benamung und Inszenierung von zunächst unbekannten Krankheitsursachen und -erregern führt, ist ebenso zu untersuchen wie die mit seinem Auftreten einsetzenden kulturell bedingten Gegenmaßnahmen.

- Effekte einer weltweiten Verdichtung und damit einer weltweiten Neugestaltung und Störung von Biosphären durch die Globalisierung sind ebenso in eine Geschichtsschreibung einzuschließen wie technische Mittel der Produktion und Kommunikation.

Diese empirischen Ansätze können - notwendig immer im Gesamtzusammenhang gesehen - in weiteren Schritten auf einzelne Regionen, Krankheiten oder weitere definierte Fragestellungen hin spezifiziert werden. Diese Liste schließt ebenso Techniken der Inszenierung von Natur, wie sie etwa mit der genetischen Sequenziertechnik in Erscheinung getreten sind, wie auch subjektive wirtschaftliche oder politische Interessen ein. Der globale Ansatz erschließt sich dadurch, dass ein Seuchengeschehen nicht etwa welthistorisch in gleicher Weise die gesamte Erde einnimmt, sondern dass ein spezifiziertes Geschehen in seinem globalen Zusammenwirken untersucht und dargestellt wird.

\subsection{Fazit: Globale und vernetzte Geschichte}

In einer globalen Seuchengeschichte können diese empirischen Ansätze jeweils Knoten in einem nicht gewichteten Netz von interagierenden Faktoren sein. 1986 hat der Technikhistoriker Thomas Hughes die Idee des „seamless web“, des übergangs- oder nahtlosen Netzes, ins Spiel gebracht. Mit diesem Ansatz ging es ihm darum, der Technikgeschichtsschreibung ein Instrument an die Hand zu geben, das sie von einer Fokussierung auf soziale Konstruktionen einerseits und von einem essenzialisierenden Biologismus sowie einer Konzentration auf Technik als angewandtes Wissen andererseits befreit (Hughes, 1986). Sein Vorschlag war, unter Bezugnahme auf Bruno Latours Akteur-Netzwerk-Theorie verschiedenste Zugangsweisen zu einer (Technik-)Geschichte als nahtlos ineinandergreifende und miteinander agierende Akteure zu analysieren. Jüngst hat Karen Kastenhofer den Wert dieses Ansatzes am Beispiel des Next-Generation-Sequencing in der Covid-19-Diagnostik exemplifiziert (Kastenhofer, 2021). 
Diese Idee möchten wir aufgreifen, dabei aber tatsächlich weniger einzelne menschliche Akteure oder nichtmenschliche Entitäten oder Objekte als Aktanten in den Mittelpunkt stellen. Vielmehr schlagen wir vor, den Zugang zu einer globalen Seuchengeschichte eher als netzwerkartig aufgebauten Ansatz zu begreifen, der keinen konkreten Anfang und kein Ende, aber auch keine führenden oder zu vernachlässigenden, sondern erst einmal gleichwertige Knoten hat, deren Gewichtungsverhälnisse sich dynamisch verschieben können. Die Knoten wiederum könnten den oben vorgeschlagenen drei Ansätzen folgen und entsprechende Themenbereiche, als gebündelte Themen wiederum Sphären konturieren. So kommen wir von Netzwerken, die Wissensentwicklungen als soziale Anerkennungsprozesse sichtbar machen (Fangerau, 2013), zu multimodalen Netzwerken, die Beziehungen und Einflüsse verschiedener Betrachtungsebenen in die Erklärung von Seuchengeschehen mit einbeziehen. Das bedeutet nicht, dass Kategorien der Komplexitätsreduktion oder erprobte denkökonomische Systematisierungen gänzlich aufgegeben werden sollen. Lebenswelt, das Virus als Aktant, Medien, Wissenschaft, Technologien, darunter selbstredend für unsere Zeit die Gentechnologie, Verwaltungshandeln, Ökonomie - alle diese Einstiegspunkte in eine sinngebende Geschichtsschreibung können erhalten bleiben. Sie sind aber als gleichwertige ungewichtete Knoten und ungerichtete Kanten des Netzwerks zu begreifen, das sich zwischen den oben vorgeschlagenen Zugangswegen zu einer globalen Seuchengeschichte aufspannt und dessen Teile nahtlos miteinander in einem zeitlich immer neu konturierten Gleichgewicht interagieren.

\subsection{Literaturverzeichnis}

Ali, S. H./Keil, R. (2008): Networked disease: Emerging infections in the global city. Blackwell, Oxford.

Bergdolt, K. (Hrsg.) (1989): Die Pest 1348 in Italien: fünfzig zeitgenössische Quellen. Manutius, Heidelberg.

Bergdolt, K. (1994): Der Schwarze Tod in Europa. Die Große Pest und das Ende des Mittelalters. Beck, München.

Crosby, A. W. (1972): The Columbian exchange: Biological and cultural consequences of 1492. Greenwood, Westport.

Diamond, J. (1997): Guns, germs, and steel. The fates of human societies. Norton, New York. 
Fangerau, H. (2013): Evolution of knowledge from a network perspective: Recognition as a selective factor in the history of science. In: Fangerau, H. et al. (Hrsg.): Classification and evolution in biology, linguistics and the history of science. Concepts - methods - visualization. Steiner, Stuttgart: 11-32.

Fangerau, H./Labisch, A. (2020): Pest und Corona. Pandemien in Geschichte, Gegenwart und Zukunft. Herder, Freiburg.

Fangerau, H. et al. (2009): Vernetztes Wissen: Kognitive Frames, neuronale Netze und ihre Anwendung im medizinhistorischen Diskurs. In: Fangerau, H./Halling, T. (Hrsg.): Netzwerke. Allgemeine Theorie oder Universalmetapher in den Wissenschaften? Ein transdisziplinärer Überblick. Transcript, Bielefeld: 29-48.

Fleck, L. (1980): Entstehung und Entwicklung einer wissenschaftlichen Tatsache. Einführung in die Lehre vom Denkstil und Denkkollektiv. Suhrkamp, Frankfurt am Main.

Garrett, L. (1994): The coming plague. Newly emerging diseases in a world out of balance. Farrar, New York.

Gehlen, A. (1956): Urmensch und Spätkultur. Philosophische Ergebnisse und Aussagen. Athenäum, Heidelberg.

Harrison, M. (2015): Reframing the history of health, medicine, and disease. In: Bulletin of the History of Medicine 89(4): 639-689.

Honigsbaum, M. (2020): The pandemic century. A history of global contagion from the Spanish Flu to Covid-19. Penguin, Cambridge.

Hughes, T. P. (1986): The seamless web: Technology, science, etcetera, etcetera. In: Social Studies of Science 16(2): 281-292.

Kastenhofer, K. (2021): The seamless web of next generation sequencing and Covid-19. In: TATuP 30(2): 18-23. Unter: https://www.tatup.de/index.php/tatup/article/view/6894 [27.10.2021].

Labisch, A. (1992): Homo hygienicus. Gesundheit und Medizin in der Neuzeit. Campus, Frankfurt am Main.

Labisch, A. (2018): Molecular historiography - neue Gegenstände und neue Methoden einer neuen Geschichtsschreibung? In: NTM Zeitschrift für Geschichte der Wissenschaften, Technik und Medizin 26: 1-16.

Latour, B. (1995): Wir sind nie modern gewesen: Versuch einer symmetrischen Anthropologie. Akademie-Verlag, Berlin.

Latour, B. (2002): Die Hoffnung der Pandora: Untersuchungen zur Wirklichkeit der Wissenschaft. Suhrkamp, Frankfurt am Main.

Le Roy Ladurie, E. (1973): Un concept: l'unification microbiénne du monde (XIV.-XVII. siècles). In: Schweizerische Zs. für Geschichte 23: 627-696. 
Martin, M./Fangerau, H. (2021): Evidenzen der Bilder. Visualisierungsstrategien in der medizinischen Diagnostik um 1900. Steiner, Stuttgart.

Matthews, A. (2020): Review of Mark Honigsbaum (2020). The pandemic century. A history of global contagion from the Spanish Flu to Covid-19. In: Postdigital Science and Education 2: 1006-1014. DOI: 10.1007/s42438-020-00170-z.

McNeill, W. H. (1976): Plagues and peoples. Anchor, New York.

Meier, M. (2020): The ,Justinianic Plague‘: An „Inconsequential Pandemic“? A Reply. In: Medizinhistorisches Journal 55(2): 172-199.

Mordechai, L. et al. (2020): Quantitative analysis and plagued assumptions: A response to Mischa Meier. In: Medizinhistorisches Journal 55(3): 290-293.

Osterhammel, J. (2009): Die Verwandlung der Welt. Eine Geschichte des 19. Jahrhunderts. Beck, München.

Quammen, D. (2012): Spillover animal infections and the next human pandemic. Norton, New York.

Rosen, G. (1947): What is social medicine? A genetic analysis of the concept. In: Bulletin of the History of Medicine 21: 674-733.

Rosenberg, C. (1989): Disease in history: Frames and framers. In: The Milbank Quarterly 67(S1): 1-15.

Rosenberg, C. (1992): Introduction. Framing disease: Illness, society, and history. In: Rosenberg, C./ Golden, J. (Hrsg.): Framing disease. Studies in cultural history. Rutgers University Press, New Brunswick: xiii-xxvi.

Rothschuh, K. E. (1978): Konzepte der Medizin in Vergangenheit und Gegenwart. Hippokrates, Stuttgart.

Spyrou, M. A. et al. (2016): Historical Y. pestis genomes reveal the European Black Death as the source of ancient and modern plague pandemics. In: Cell Host Microbe 19(6): 874-881.

Viglione, G. (2020): How many people has the coronavirus killed? In: Nature 585(7823): 22-24. 